\title{
THE LEVITZKI RADICAL IN JORDAN RINGS
}

\author{
CHESTER E. TSAI
}

The main purpose of this paper is to give an external characterization of the Levitzki radical of a Jordan ring $\mathfrak{A}$ as the intersection of a family of prime ideals $\mathfrak{A}$. This characterization coincides with that of associative rings which was given by Babic in [1].

Applying this characterization, it is easy to see that the Levitzki radical of a Jordan ring contains the prime radical of the same ring. For associative rings the same statement is well known, since the prime radical in associative rings is called the Baer radical. If the minimal condition on ideals holds on Jordan ring $\mathfrak{A}$, then the Levitzki radical, $L(\mathfrak{A})$, and the prime radical, $R(\mathfrak{A})$ of $\mathfrak{A}$ coincide.

Throughout this paper, any Jordan ring $\mathfrak{A}$, that is a (nonassociative) ring satisfying

(1) $a b=b a$, and

(2) $a^{2}(a b)=a\left(a^{2} b\right)$ for all $a, b$ in $\mathfrak{A}$, and any of its subrings satisfy the conditions,

(3) $2 a=0$ implies $a=0$ and

(4) if $a$ is in a subring $C$ of $\mathfrak{A}$ then there exists a unique element $x$ in $C$ such that $2 x=a$.

In a Jordan ring, the following identity $\left({ }^{*}\right)$ is well known. One can find the proof in $[3]$.

$$
R_{(x y) z}=R_{x} R_{y z}+R_{y} R_{z x}+R_{z} R_{x y}-R_{x} R_{z} R_{y}-R_{y} R_{z} R_{x}
$$

where, for any element $u$ in $\mathfrak{A}, R_{u}$ is the formal multiplication of $u$ in the ring $\mathfrak{A}$, i.e., $a R_{u}=a u$ for all $a$ in $\mathfrak{A}$.

If $A, B$ are subsets of $\mathfrak{A}, A U_{B}$ denotes the set of finite sums of elements of the form $a U_{b}$ where $a$ is in $A$ and $b$ is in $B$ and $a U_{b}=2(a b) b-a b^{2}$. If $A$ and $B$ are ideals in $\mathfrak{A}$, then $A^{3},(A B) B+A(B B)$ and $A U_{B}$ are ideals in $\mathfrak{A}$. The first two are proved in [5]. To prove $A U_{B}$ is an ideal, let $a$ be an element in $A$ and $b, c$ be elements in $B$ and $a U_{(b, c)}$ $=\frac{1}{2} a\left(U_{b+c}-U_{b}-U_{c}\right)=(a b) c+(a c) b-(b c) a$. Assume that both $A$ and $B$ are ideals in $\mathfrak{A}$, and applying $\left({ }^{*}\right)$ one can easily check that for any $u$ in $\mathfrak{A},\left(a U_{(b, c)}\right) \cdot u=a U_{\left(b^{\prime}, c\right)}+a U_{\left(b, c^{\prime}\right)}-a^{\prime} U_{(b, c)}$ where $a^{\prime}=a u, b^{\prime}=b u$, and $c^{\prime}=c u$. Letting $c=b$ one sees that $A U_{B}$ is an ideal in $\mathfrak{A}$ if $A$ and $B$ are. It is proved in [4] that if $A$ is an ideal in $\mathfrak{A}$, then $A^{3}=A U_{A}$.

If $\mathfrak{A}$ is a Jordan ring, we denote $\mathfrak{A}^{3}$ by $\mathfrak{A}_{1}$, and, inductively $\mathfrak{A}_{i+1}=\mathfrak{A}_{i}^{3}$ for each $i$. We also denote $\mathfrak{A}_{(0)}$ as $\mathfrak{A}$ and, inductively $\mathfrak{A}_{(i+1)}=\mathfrak{A}_{(i)} \mathfrak{A}_{(i)}$

Received by the editors November 25, 1968 and, in revised form, May 20, 1969. 
for each $i$. The ring $\mathfrak{A}$ is said to be nilpotent if there exists a positive integer $k$ such that $\mathfrak{A}^{k}=0$, where $\mathfrak{A}^{k}$ is the set of all finite sums of all products of $k$ elements from $A$, under any association, and is said to be solvable if there exists a positive integer $t$ such that $\mathfrak{A}_{(t)}=0$. It is clear if $\mathfrak{A}$ is nilpotent then $\mathfrak{A}$ is solvable. It is also clear that $\mathfrak{A}_{m} \subseteq \mathfrak{A}_{(m)}$ for all $m$. Note that for each $m \mathfrak{A}_{m}$ is an ideal in $\mathfrak{A}$.

Lemma A. Let $\mathfrak{A}$ be a Jordan ring which is generated by a set of $n$ generators. Then there exists a natural number $f(n, m)$ so that $\mathfrak{A}^{(n, m)}$ $\subseteq \mathfrak{A}_{m}$.

This lemma is a key lemma in the paper [5]. Since the proof is lengthy we shall not provide it here.

THEOREM B. If $\mathfrak{A}$ is a Jordan ring which is finitely generated then $\mathfrak{A}$ is nilpotent if $\mathfrak{A}$ is solvable.

Proof. $\mathfrak{U}^{f(n, m)} \subseteq \mathfrak{A}_{m} \subseteq \mathfrak{A}_{(m)}$.

Definition 1. A Jordan ring $\mathfrak{A}$ is locally nilpotent if every finitely generated subring is solvable.

Corollary (to Theorem $\mathrm{B}$ ). Let $\mathfrak{A}$ be a Jordan ring. Then $\mathfrak{A}$ is locally nilpotent if and only if $\mathfrak{A}$ is locally solvable.

Lemma C. Let $\mathfrak{A}$ be a finitely generated Jordan ring. Then, for any natural number $m, \mathfrak{A}_{m}$ is also finitely generated.

This lemma is given in [5]. However, since it is needed in the proof of Theorem 1 of this article, we shall give the proof of the lemma here.

Proof. If $X=\left\{x_{1}, x_{2}, \cdots, x_{n}\right\}$ is a set of generators of $\mathscr{H}$ let $Y$ be the set of all commutative (but not associative) words of $x$ 's which are in $\mathfrak{A}_{1}$ and which are of $X$-length less than $f(n, 2)$, where $f(n, 2)$ is the natural number such that $\mathfrak{H}^{f(n, 2)} \subseteq \mathfrak{A}_{2}=\left(\mathfrak{A}_{1} \mathfrak{A}_{1}\right) \mathfrak{H}_{1}$. Since $X$ is a finite set, $Y$ is a finite subset in $\mathfrak{A}_{1}$. We want to show that $\mathfrak{A}_{1}$ is generated by $Y$, thus $\mathfrak{A}_{1}$ is finitely generated. The proof of the lemma is then completed by induction on $m$.

A monomial $m=x_{i_{1}} x_{i_{2}} \cdots x_{i_{k}} ; i_{j}=1,2, \cdots, n$ is said to be of length $k$, which is denoted by $l(m)=k$, regardless of what is the association. If $a$ is an element in $\mathfrak{A}_{1}=(\mathfrak{H} \mathfrak{H}) \mathfrak{A}$, then there exist monomials $a_{i}, b_{i}, c_{i}$ such that $a=\sum_{i=1}^{t}\left(a_{i} \cdot b_{i}\right) \cdot c_{i}=u_{1}+u_{2}+\cdots+u_{i}$. Noted $u_{i}$ itself is also an element of $\mathfrak{A}_{1}$ for each $i$.

If $u$ is one of those $u_{i}$ 's and $l(u) \geqq f(n, 2)$ then, by Lemma A, $u \in \mathscr{A}_{2}$ $=\left(\mathfrak{H}_{1} \mathfrak{A}_{1}\right) \mathfrak{H}_{1}$, i.e. $u=\sum_{i}\left(r_{i} \cdot s_{i}\right) \cdot t_{i}$ where $r_{i}, s_{i}, t_{i} \in \mathfrak{A}_{1}$ and $l\left(r_{i}\right)+l\left(s_{i}\right)$ $+l\left(t_{i}\right)=l(u)$. We could repeat the process on $r_{i}, s_{i}$ and $t_{i}$. Thus we may assume $l(u)<f(n, 2)$. But that is to say $u$ is a member of $Y$ and 
$a$ is an element in the subalgebra of $\mathfrak{A}_{1}$ which is generated by $Y$. Thus $Y$ generates $\mathfrak{A}_{1}$.

Induction on $m$ can be easily carried out.

Definition 2. Let $\mathfrak{A}$ be a Jordan ring. An ideal $I$ in $\mathfrak{A}$ is a locally nilpotent (locally solvable) ideal if $I$, considered as a ring itself, is locally nilpotent (locally solvable).

Lemma D. Let I be a locally solvable ideal in a Jordan ring $\mathfrak{A}$. If the factor ring $\overrightarrow{\mathfrak{A}}=\mathfrak{A} / I$ is also locally solvable, then $\mathfrak{A}$ is locally solvable.

Lemma E. The sum of all locally solvable ideals in a Jordan ring $\mathfrak{A}$ is a locally solvable ideal in $\mathfrak{A}$.

Definition 3. The maximal locally solvable ideal, $L(\mathfrak{A})$, of a Jordan ring $\mathfrak{A}$ is called the Levitzki radical of $\mathfrak{A}$.

Definition 4. A Jordan ring $\mathfrak{A}$ is Levitzki-semisimple (or $L$-semisimple) if the Levitzki radical of $\mathfrak{A}$ is 0 , i.e., $L(\mathfrak{H})=0$.

TheOREM F. Every Jordan ring $\mathfrak{A}$ has a Levitzki radical. The factor ring $\mathfrak{A} / L(\mathfrak{H})$ of $\mathfrak{A}$ over its Levitzki radical $L(\mathfrak{U})$ is Levitzki-semisimple.

Lemmas $\mathrm{D}, \mathrm{E}$, and Theorem $\mathrm{F}$ are given in [5]. The proofs are similar to the corresponding statements in associative rings.

Recently Babic gave (in [1]) an external characterization of the Levitzki radical, $L(R)$, for an associative ring $R$ as the intersection of the family of prime ideals $\left\{P_{i}\right\}$ in $R$ whose factor rings $\left\{R / P_{i}\right\}$ are Levitzki-semisimple. We shall show the same characterization holds for Jordan rings.

Definition 5. An ideal $P$ in $\mathfrak{A}$ is a prime ideal if whenever $A, B$ are ideals in $\mathfrak{A}$ such that $A U_{B} \subseteq P$ then either $A \subseteq P$ or $B \subseteq P$.

THEOREM 1. The Levitzki radical $L$ of a Jordan ring $\mathfrak{A}$ is the intersection of all prime ideals $P_{\alpha}$ of $\mathfrak{A}$ for which $\mathfrak{A} / P_{\alpha}$ is Levitzki-semisimple.

Proof. Let $P=\cap P_{\alpha}$, where the intersection is taken over all prime ideals $P_{\alpha}$ of $\mathfrak{A}$ for which $\mathfrak{A} / P_{\alpha}$ is Levitzki-semisimple.

For each $P_{\alpha}, L \subseteq P_{\alpha}$, otherwise $L+P_{\alpha} / P_{\alpha} \cong L / L \cap P_{\alpha}$ is a nonzero locally nilpotent ideal in the $L$-semisimple ring $\mathfrak{A} / P_{\alpha}$. Thus $L \subseteq \bigcap_{\alpha} P_{\alpha}$ $=P$.

It remains to show that $P \subseteq L$. If $x \notin L$, then $x \notin Q$ where $Q$ is a prime ideal of $\mathfrak{A}$ such that $\mathfrak{A} / Q$ is $L$-semisimple. Since $x \notin L$, the principal ideal $(x)$ generated by $x$ is not locally nilpotent, in other words, there exists a finitely generated subring $S$ (generated by $n$ elements) of $(x)$ which is not nilpotent. By Lemma C, for any integer 
$m, S_{m}$ is finitely generated. Hence $S_{m}$ is not nilpotent, otherwise $S^{f(n, m)} \subseteq S_{m}$ forces $S$ to be nilpotent, which is a contradiction.

Let $\subseteq$ be the collection of all ideals $Q$ in $\mathfrak{A}$ such that $S_{m} \Phi Q$ for all positive integers $m$. The set $\subseteq$ is not empty and can be ordered by inclusion. We shall apply Zorn's lemma to obtain an ideal $Q$ which is a maximal element in $\mathfrak{S}$. For this, it is only necessary to verify that the union of an ascending chain $\left\{Q_{i}\right\}$ in $\subseteq$ is again in $\subseteq$.

Let $Q_{1} \subseteq Q_{2} \subseteq Q_{3} \subseteq \ldots$ be an ascending chain of elements in $\subseteq$ and let $Q^{*}=\cup_{i} Q_{i}$. Clearly $Q^{*}$ is an ideal in $\mathfrak{A}$. Now if $S_{m} \subseteq Q^{*}$ for some integer $m$, then $S_{m} \subseteq Q_{j}$ for some $j$, since $S_{m}$ is finitely generated. But this is impossible since $S_{m} \subseteq Q_{j}$ means $Q_{j} \notin \subseteq$. Thus we have shown that $Q^{*}$ is in $\subseteq$.

If $Q$ is an ideal in $\mathfrak{A}$ which is a maximal element in $\mathfrak{S}$, then $Q$ is a prime ideal in $\mathfrak{A}$. Suppose this is not true, then there exist ideals $A^{\prime}$, $B^{\prime}$ of $\mathfrak{A}$ such that $A^{\prime}, B^{\prime}$ are not contained in $Q$ but $A^{\prime} \bigcup_{B^{\prime}}$ is contained in $Q$. The ideals $A=A^{\prime}+Q$ and $B=B^{\prime}+Q$ both properly contain $Q$. Thus, by the maximality of $Q, A$ and $B$ are not members of $\mathfrak{S}$. Hence, there exist integers $n$ and $m$ such that $S_{n} \subseteq A$ and $S_{m} \subseteq B$. Without loss of generality, we may assume $n \leqq m$. Then, since $S_{m} \subseteq S_{n}, S_{m}$ is contained in both $A$ and $B . S_{m+1}=\left(S_{m} S_{m}\right) S_{m}=S_{m} \bigcup_{S_{m}} \subseteq A \cup_{B} \subseteq A^{\prime} \cup_{B^{\prime}}$ $+Q=Q$. This is impossible.

We now show that the factor ring $\mathfrak{A} / Q$ is Levitzki-semisimple. Let $\bar{N}=N / Q$ be a locally nilpotent (solvable) ideal of $\overline{\mathfrak{A}}=\mathfrak{A} / Q$. If $\bar{N} \neq 0$, then we know that $N \geqq Q$ and thus for some $m, N \geqq S_{m}$. Then $\bar{S}_{m}$ is a finitely generated subring of the locally nilpotent ring $\bar{N}$. Therefore $\bar{S}_{m}$ is nilpotent, $\left(\bar{S}_{m}\right)_{n}=0$ and thus $S_{m+n} \subseteq Q$, a contradiction.

So far we have found a prime ideal $Q$ for which $\mathfrak{A} / M$ is $L$-semisimple. Thus the proof of the theorem is completed if we show $x \notin Q$. But it is clearly so, for if $x \in Q$ then $S$, which is a subring of $(x)$, will be contained in $Q$, which is a contradiction.

THEOREM 2. Every L-semisimple ring $\mathfrak{A}$ is isomorphic to a subdirect sum of prime L-semisimple rings.

Since the ring $\mathfrak{A}$ is Levitzki-semisimple the intersection of the family $\left\{P_{i} \mid i \in I\right\}$ of prime ideals such that $\mathfrak{A} / P_{\boldsymbol{i}}$ is $L$-semisimple is zero. The ring $\mathfrak{A}$ is isomorphic to the subdirect sum of the family $\left\{S_{i} \mid i \in I\right\}$ of prime $L$-semisimple rings, where $S_{i} \cong \mathfrak{U} / P_{i}$ for all $i \in I$.

In [4], the prime radical $R(\mathfrak{H})$ of a Jordan ring is defined to be the prime radical of the zero ideal in $\mathfrak{A}$. For any ideal $I$ in $\mathfrak{A}$, the prime radical $I^{Q}$ was shown to be equal to the intersection of all prime ideals in $\mathfrak{A}$ containing $I$. Thus $R(\mathfrak{U})$ is the intersection of the family of all prime ideals in $\mathfrak{A}$, and we have 
THEOREM 3. For any Jordan ring the prime radical $R(\mathfrak{A})$ is contained in the Levitzki radical $L(\mathfrak{A})$.

However, in the presence of the minimal condition for ideals in $\mathfrak{A}$ we find in [5] the following theorem:

Theorem G (Theorem 3 in [5]). If $\mathfrak{A}$ is a locally solvable Jordan algebra over a field $F$ (or a Jordan ring) in which minimal conditions on ideals hold, then $\mathfrak{A}$ is nilpotent.

Now if $\mathfrak{A}$ is a Jordan ring in which minimal conditions on ideals hold then the same condition holds for the factor ring $\mathfrak{A} / R(\mathfrak{A})$. Thus the Levitzki radical $L(\mathfrak{U} / R(\mathfrak{H}))$ of $\mathfrak{A} / R(\mathfrak{H})$ is a nilpotent ideal in $\mathfrak{A} / R(\mathfrak{A})$. However, Theorem 8 of [4] stated that a Jordan ring $J$ is $Q$-semisimple, (i.e., $R(J)=0$ ) if and only if it contains no nonzero nilpotent ideals. Thus it amounts to say $L(\mathfrak{H} / R(\mathfrak{A}))=0$, i.e., $\mathfrak{A} / R(\mathfrak{U})$ is $L$-semisimple. We have proved:

THEOREM 4. If $\mathfrak{A}$ is a Jordan ring in which the minimal condition on ideals holds, then the Levitzki radical, $L(\mathfrak{H})$, and the prime radical, $R(\mathfrak{H})$, of $\mathfrak{A}$ coincide.

CoRollary. If $\mathfrak{A}$ is a Jordan ring satisfying D.C.C. on quadratic ideals then $L(\mathfrak{H})=R(\mathfrak{H})$.

\section{REFERENCES}

1. A. M. Babic, The Levitzki radical, Dokl. Akad. Nauk. SSSR 126 (1959), 242243. (Russian)

2. N. J. Divinsky, Rings and radicals, University of Toronto Press, Toronto, Canada, 1965.

3. R. D. Schafer, An introduction to non-associative algebras, Academic Press, New York, 1966.

4. C. E. Tsai, The prime radical in a Jordan ring, Proc. Amer. Math. Soc. 19 (1968), 1171-1175.

5. K. A. Ževlakav, Solvability and nilpotence of Jordan rings, Algebra i Logika Sem. 5 (1966), 37-58. (Russian)

Michigan State University 